\title{
Gewerbliche Hochtechnologieausbildung für die Sensorik-Branche: Berufe und Bedarfe
}

\author{
Globisch, Sabine \\ VDI/VDE Innovation + Technik GmbH \\ Steinplatz 1 \\ 10623 Berlin
}

Diskussionen um den drohenden oder bereits spürbaren Fachkräftemangel begleiten die technologische Entwicklung in den Schlüsseltechnologien Biotechnologie, Mikrotechnologie, Nanotechnologie und optische Technologie in Deutschland bereits seit über einem Jahrzehnt. Dabei beziehen die Diskussion die gewerbliche und die akademische Ebene in gleicher Weise ein, denn es wird ein Mangel an ausgebildeten Ingenieuren genauso beklagt wie die fehlende Zahl an gewerblichen Fachkräften.

Die Versorgung mit ausreichendem Fachpersonal gilt als ein Schlüssel für den erfolgreichen Zugang zu den zahlreichen Märkten. Die erwarteten Qualifikationsprofile bei den Fachkräften sind so breit gefächert wie es die Spezialisierungen der Unternehmen, bzw. die Branchenspezifika der verschiedenen Anwendungsbereiche der Schlüsseltechnologien auch sind. So werden in einem Unternehmen Feinoptiker beschäftigt, wo ein anderes Unternehmen eine Mikrotechnologin eingestellt hat, weil am entsprechenden Arbeitsplatz Reinraumerfahrung erforderlich ist.

Aus der Arbeit in den Aus- und Weiterbildungsnetzwerken für die Mikrosystemtechnik sind diese Vorgehensweisen im Umgang mit diesen Herausforderungen des Fachkräftemangels, den Qualifikationsbedarfen und Berufen bekannt. Offen war bislang jedoch die Fragen, vor welchen Herausforderungen die Unternehmen aus der Sensorik und Messtechnik stehen, wenn es um ihren Fachkräftebedarf geht.

Aus den Unternehmen der Mikrosystemtechnik der unterschiedlichen Regionen ${ }^{1}$ ist bekannt, dass sie eine Reihe verschiedener Berufe ausbilden, weil die vermittelten Kompetenzen in den Ausbildungsberufen sich in wesentlichen Lernfeldern ergänzen oder dass diese Unternehmen gar nicht ausbilden, weil sie nicht entscheiden können, welcher der Ausbildungsberufe am besten geeignet ist in Bezug auf die Anforderungen im Unternehmen.

Die Studie, die bei Unternehmen der Sensorik und Messtechnik (mit Unterstützung des AMA Fachverbandes für Sensorik) zwischen Juni 2007 und Mai $2008^{2}$ durchgeführt wurde ergab, dass diese Unternehmen auf der gewerblichen Ebene hauptsächlich an Fachkräften aus den Berufen Mechaniker, Elektroniker, Mechatroniker, Mikrotechnologe interessiert sind.

\footnotetext{
${ }^{1}$ Informationen liegen aus Niedersachsen und Thüringen vor.

${ }^{2}$ Künzel, Matthias; Hartmann, Ernst A.: Technische Fach- und Führungskräfte in der Sensorik und Messtechnik, Berlin (im Druck).
} 
Die Verfügbarkeit von Fachkräften hat sich in den letzten fünf Jahren...

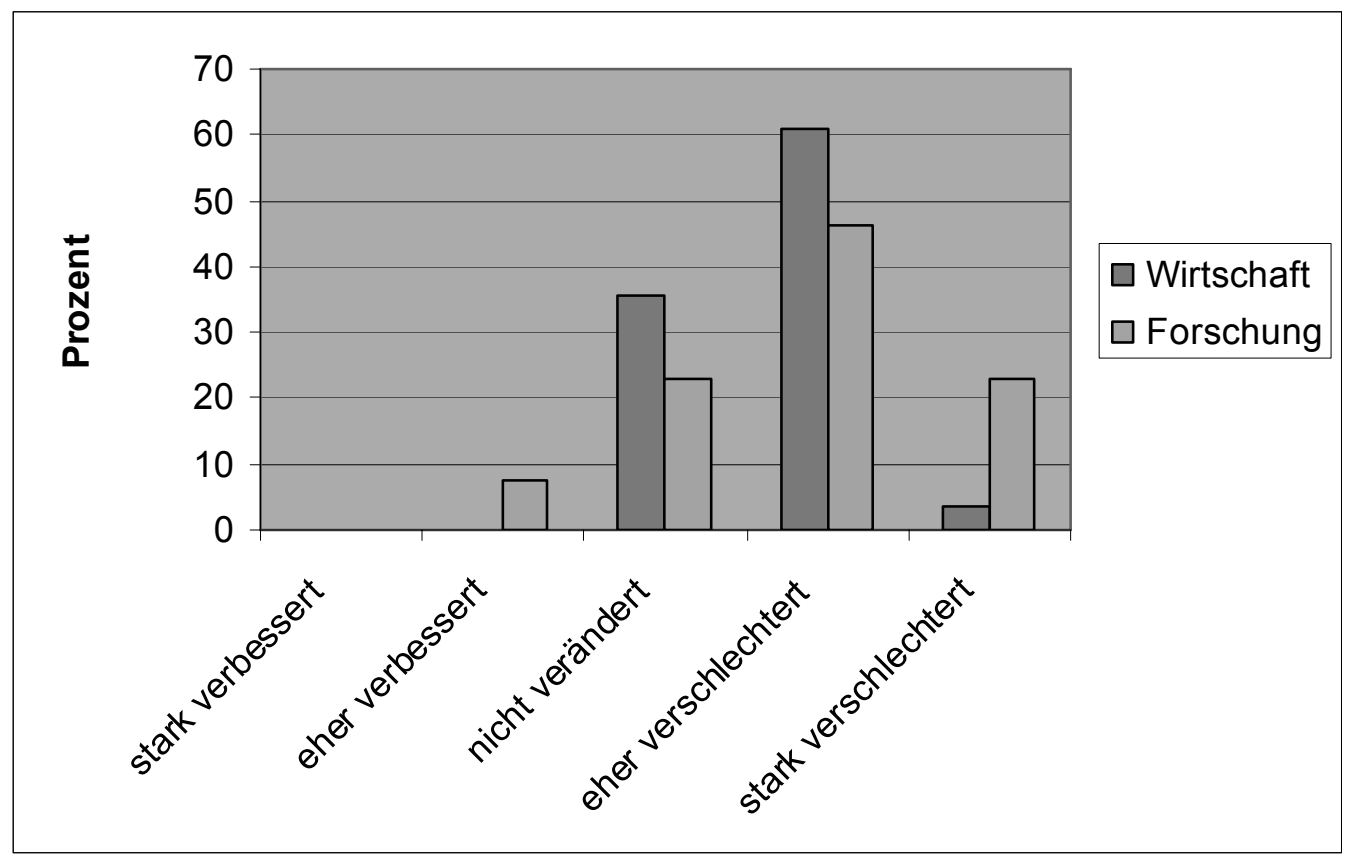

Allerdings müssen die Unternehmen der Sensorik und Messtechnik noch andere Strategien zur Bewältigung des gefühlten und gefürchteten Fachkräftemangels nutzen. Auf Basis der Aussagen von 64 Industrieunternehmen und 13 Forschungseinrichtungen ließen sich zunächst ganz typische Taktiken identifizieren. So zeichnet sich die Branche der „Hidden Champions“ mit einem Weltmarktanteil von ca. 30 Prozent durch eine überdurchschnittlich hohe Beteiligung an der gewerblichen Ausbildung aus.

Über 70 Prozent der Unternehmen sind bereits Ausbildungsbetriebe. Da sich aber der erwartete Fachkräftemangel eher auf die akademisch qualifizierten Fach- und Führungskräfte beziehen wird, müssen die Möglichkeiten der adäquaten Fachkräfterekrutierung in einem breiteren Rahmen angelegt werden.

Neben den bisherigen Strategien zur Fachkräfterekrutierung - vorwiegend durch persönliche Kontakte oder Stellenanzeigen im Internet - nutzen die Unternehmen der Sensorik und Messtechnik hauptsächlich die Vergabe von Praktikumsplätzen sowie Studien und Diplomarbeiten zur Fachkräftesicherung. Außerdem werden die bestehenden Forschungskooperationen sowohl für Ausbildungsverbünde in der gewerblichen Ausbildung genutzt als auch für die Weiterbildung des bestehenden Personals. Zu einem kleinen Teil nutzen die Unternehmen bereits die Möglichkeiten eines Dualen Studiums oder des Fernstudiums, um Fachkräfte einschlägig weiter zu qualifizieren.

Reichen diese Maßnahmen aus, um langfristige die ausreichende Zahl von Fach- und Führungskräften in der Sensorik und Messtechnik zu rekrutieren und zu halten?

Neben den Befunden aus der Studie sollen erprobte Strategien zu Bewältigung des Fachkräftemangels z. B. durch gezielte Weiterbildung vorgestellt werden. Flankierend sind allerdings auch Konzepte zur Umsetzung gewerblicher Ausbildung in den Betrieben sinnvoll. Diese werden im Rahmen der regionalen Good practice Beispiele vorgestellt. 